\title{
Recent advances in superconducting nanowire single-photon detector technology for exoplanet transit spectroscopy in the mid-infrared
}

\author{
Emma E. Wollman $\odot,{ }^{a}, *$ Varun B. Verma, ${ }^{b} *$ Alexander B. Walter, ${ }^{a}$ \\ Jeff Chiles, ${ }^{\text {b }}$ Boris Korzh, ${ }^{\text {a }}$ Jason P. Allmaras $\odot,{ }^{\text {a }}$ Yao Zhai $\odot$, b,c

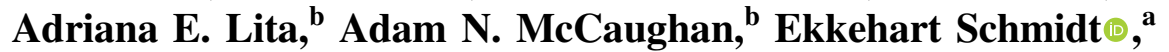 \\ Simone Frasca $\odot,{ }^{\text {a,d }}$ Richard P. Mirin, ${ }^{\text {b }}$ Sae Woo Nam, ${ }^{\text {b }}$ and \\ Matthew D. Shaw ${ }^{a}$ \\ ${ }^{a}$ Jet Propulsion Laboratory, California Institute of Technology, Pasadena, California, \\ United States \\ ${ }^{b}$ National Institute of Standards and Technology, Boulder, Colorado, United States \\ 'University of Colorado, Department of Physics, Boulder, Colorado, United States \\ dÉcole Polytechnique Fédérale de Lausanne, Advanced Quantum Architecture Laboratory, \\ Neuchâtel, Switzerland
}

\begin{abstract}
The Origins Space Telescope mission concept includes an exoplanet transit spectrometer that requires detector arrays with ultrahigh pixel-to-pixel stability. Superconducting nanowire single-photon detectors, or SNSPDs, have the potential to meet these stringent stability requirements due to their digital-like output. Traditionally used for applications at near-IR telecom wavelengths, SNSPDs have demonstrated near-unity detection efficiencies, ultralow dark-count rates, and high dynamic ranges. Until recently, however, SNSPD operation at the mid-infrared (mid-IR) wavelengths of interest for Origins had not been demonstrated, and SNSPD formats were limited to small arrays and active areas. Recent advances in SNSPD fabrication techniques have pushed SNSPD sensitivity to wavelengths beyond $7 \mu \mathrm{m}$ and have enabled millimeter-scale active areas and kilopixel arrays. We report here on this progress and the outlook toward developing arrays of ultrastable superconducting nanowire single-photon detectors for mid-IR astronomy applications. (c) The Authors. Published by SPIE under a Creative Commons Attribution 4.0 Unported License. Distribution or reproduction of this work in whole or in part requires full attribution of the original publication, including its DOI. [DOI: 10.1117/1.JATIS.7.1 $.011004]$
\end{abstract}

Keywords: superconducting nanowire single-photon detectors; superconducting detectors; single-photon detectors; infrared; detectors; space telescope.

Paper 20066SS received Jun. 9, 2020; accepted for publication Dec. 9, 2020; published online Jan. 12, 2021.

\section{Introduction}

The Origins Space Telescope (Origins) is one of the four Large Mission Concept Studies commissioned by NASA for the National Academy of Sciences' 2020 Astronomy and Astrophysics Decadal Survey. Origins traces our cosmic history, from the formation of the first galaxies and the rise of metals to the development of habitable worlds and present-day life. Origins does this through exquisite sensitivity to infrared radiation from ions, atoms, molecules, dust, water vapor, and ice, and observations of extra-solar planetary atmospheres, protoplanetary disks, and largearea extragalactic fields. Origins operates in the wavelength range 2.8 to $588 \mu \mathrm{m}$ and is more than 1000 times more sensitive than its predecessors due to its large, cold $(4.5 \mathrm{~K})$ telescope and advanced instruments.

The search for life on other planets is a central objective of NASA's astrophysics program. ${ }^{1}$ To this end, Origins will use its Mid-Infrared Spectrometer and Camera (MISC) instrument to search for biosignatures in the atmospheres of exoplanets. ${ }^{2}$ MISC includes an exoplanet transit

*Address all correspondence to Emma E. Wollman, emma.e.wollman@jpl.nasa.gov; Varun B. Verma, varun.verma@nist.gov 
(a)

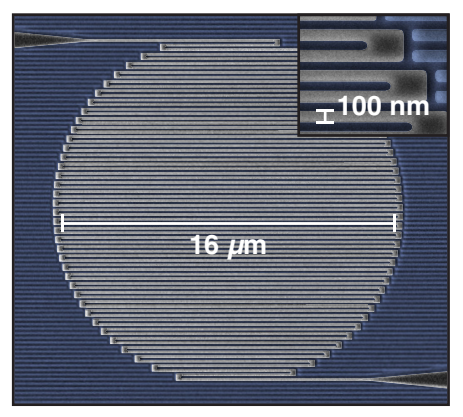

(b)

(c)
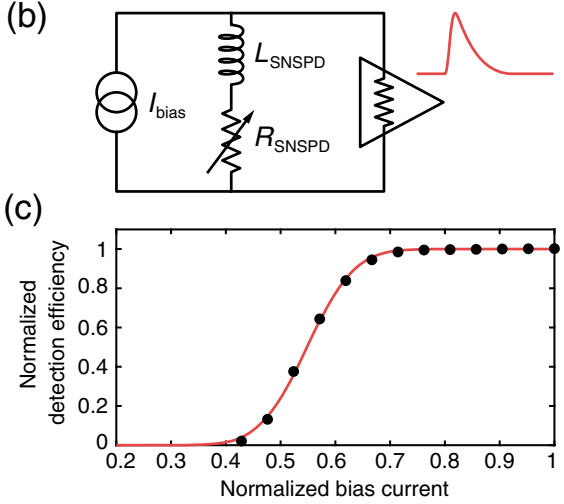

Fig. 1 (a) False-color SEM of a single-pixel SNSPD designed to couple to light from a single-mode fiber. SNSPDs are lithographically patterned from superconducting films with thicknesses on the order of a few nanometers and widths from tens to hundreds of nanometers. The nanowire is meandered to fill the desired pixel active area, and the nanowire layer can be embedded in an optical stack to enhance optical absorption into the nanowire. (b) Equivalent circuit of an SNSPD pixel. The SNSPD is treated as an inductor $\left(L_{S N S P D}\right)$ and a variable resistor $\left(R_{\mathrm{SNSPD}}\right)$ in series. Typical SNSPD inductances are on the order of $1 \mu \mathrm{H}$ due to the high kinetic inductivity in superconducting thin films. The nanowire is current-biased below its critical current and connected in parallel to a readout circuit, typically with a $50-\Omega$ input impedance. When a photon of sufficient energy is absorbed in the nanowire, it forms a local resistive region or hotspot. Joule heating from the current flowing through this region causes it to expand until the hotspot develops a resistance on the order of $R_{\text {SNSPD }}=1 \mathrm{k} \Omega$. As the nanowire resistance increases, current is diverted into the readout on the time scale of the $L_{\mathrm{SNSPD}} /\left(R_{\mathrm{SNSPD}}+50 \Omega\right)$ time constant $(\sim 1 \mathrm{~ns})$. With the current diverted, the resistive region cools off and returns to its superconducting state. Current returns to the nanowire with a time scale of $L_{S N S P D} / 50 \Omega \sim 20$ ns. (c) Typical detection efficiency versus bias current curve for an SNSPD. At sufficiently high bias currents, the efficiency reaches a plateau, indicating that the internal detection efficiency has saturated and that every absorbed photon produces a detection event. Measurements for a device at $1550 \mathrm{~nm}$ are shown with black circles and the red curve is an error function fit to the data.

spectrometer designed to detect small changes in the spectrum of a star due to the absorption or emission from the atmosphere of a transiting exoplanet at wavelengths from 2.8 to $20 \mu \mathrm{m}$. This spectral range contains numerous molecular signatures, which could be indicative of life on an exoplanet, including water, methane, ozone, carbon dioxide, and nitrous oxide. One of the key challenges for the MISC transit spectrometer is the required 5-ppm pixel-to-pixel detector stability needed to detect the minute changes in the host star's spectrum over the course of a transit. A detector's stability may be a function of the detecting element itself or of the detector's electronic readout. For infrared detectors used on previous space telescopes, it appears that drifts in the readout amplifiers limit the photometric stability to a few tens of parts per million. ${ }^{3,4}$ Further improvement of these detectors is, therefore, necessary to reach the desired stability.

With a photon-counting detector such as a superconducting nanowire single-photon counter [SNSPD—see Fig. 1(a)], the detector's output signal is essentially digital [Fig. 1(b)] and is, therefore, not affected by drifts in amplifier gain or voltage offsets that can lead to instabilities in the readout of analog signals. Instead, the dominant source of instabilities in a photon-counting detector becomes the detection efficiency itself. In SNSPDs, the probability of detecting a photon increases with increasing bias current, because the current serves to both assist and amplify the detection process. As seen in Fig. 1(c), it is possible to produce SNSPDs with an efficiency that saturates at higher bias currents, indicating that every photon absorbed in the nanowire produces a detection event. When an SNSPD is biased in this efficiency plateau region, drifts in current or temperature do not produce equivalent drifts in efficiency. It is worth noting that other superconducting detectors, such as transition edge sensors (TESs) and microwave kinetic inductance detectors (MKIDs), can also be operated as photon-counting detectors with the added benefit of energy resolution. ${ }^{5,6}$ However, the recovery time after a detection event for TESs and MKIDs is 2 to 4 orders of magnitude slower than is typical for SNSPDs. This reduced count rate dynamic range will cause TESs and MKIDs to have difficulty keeping up with the 
photon fluxes expected from the MISC transit spectrometer unless arrays with 100 to 10,000 times more pixels are used. Additionally, the spectral response of TESs and MKIDs is sensitive to changes in operating temperature, bias (TESs), and rf pickup (MKIDs). TESs and MKIDs use the spectral response to discriminate between low-energy photons and readout noise. SNSPDs are thus uniquely promising candidates for MISC.

Although SNSPDs may be able to meet MISC's key requirement of 5-ppm stability, the large-format arrays of mid-IR SNSPDs necessary for MISC do not exist at this time. In order to produce the required ultrastable SNSPD arrays, two major technology challenges must first be addressed: (1) obtaining saturated efficiency in the mid-IR and (2) yielding millimeter-scale arrays with kilopixel formats. SNSPDs are a relatively young technology, and so far, most technology development has been focused at telecom wavelengths, where SNSPDs have been shown to have detection efficiencies above $95 \%,{ }^{7}$ intrinsic dark count rates well below $1 \mathrm{count} / \mathrm{hr} /$ pixel $^{,}{ }^{8}$ and maximum count rates over $50 \mathrm{MHz}$ per pixel. ${ }^{9}$ For mid-IR applications, saturated efficiency had been demonstrated to $5 \mu \mathrm{m},{ }^{10}$ but it is only recently that progress has been made on optimizing SNSPDs for wavelengths beyond $5 \mu \mathrm{m} .{ }^{11,12}$ Until recently, the largest existing near-IR SNSPD arrays had a 300- $\mu \mathrm{m}$ diameter and consisted of 64 pixels. ${ }^{13}$ In the past year, groups have separately demonstrated a single pixel with a $400 \times 400 \mu \mathrm{m}^{2}$ active area ${ }^{8}$ and a kilopixel array with a $1.6 \times 1.6 \mathrm{~mm}^{2}$ area, of which $960 \times 960 \mu \mathrm{m}^{2}$ consisted of active elements. ${ }^{14}$

In this paper, we discuss the suitability of using SNSPDs for the MISC instrument on Origins. Section 2 is a review of recent laboratory advances in mid-IR sensitivity, detector array multiplexing, and array active area. In Sec. 3, we present a new analysis of SNSPD stability.

\section{Advances in SNSPD Technology}

\subsection{Mid-IR Detection}

The latest design of the MISC transit spectrometer includes three different wavelength bands covering the range from 2.8 to $20 \mu \mathrm{m}$ : TRA-S from 2.8 to $5.5 \mu \mathrm{m}$, TRA-M from 5.5 to $11 \mu \mathrm{m}$, and TRA-L from 10.5 to $20 \mu \mathrm{m}^{2}$. The current baseline is to use HgCdTe detectors for the shorter bands and blocked impurity band detectors (BIBs) for TRA-L. Recent measurements of Si:As BIBs show that they should be able to meet the TRA-L stability requirement of $20 \mathrm{ppm},{ }^{15}$ but no detector to date has demonstrated the stringent $<5$-ppm stability required for the TRA-S and TRA-M bands. Recent advances in SNSPD technology indicate that it should be possible to achieve saturated internal detection efficiency out to $11 \mu \mathrm{m}$ in the near future, making SNSPDs a possible candidate for these bands. A demonstration of SNSPD sensitivity out to $20 \mu \mathrm{m}$ has yet to be attempted and remains an open question in the field.

In order to improve the energy sensitivity of the nanowire detectors and extend the photoresponse out to longer wavelengths, there are several possible approaches. One is to decrease the cross-sectional area of the nanowire. This results in a higher probability of a hotspot being generated since the energy per unit area is higher and thermal conductivity along the length of the nanowire is smaller. Another approach is to engineer the material to reduce the superconducting gap energy. Reducing the superconducting gap energy results in a larger number of broken Cooper pairs (quasiparticles) for a given amount of deposited energy in the superconductor. Alternatively, the material can be engineered to reduce the free carrier density or density of states. Reducing the free carrier density or increasing the resistivity of the material results in the deposited energy being divided among fewer quasiparticles, thus increasing the effective temperature of each quasiparticle.

The first approach, reducing the width or thickness of the nanostrip, is challenging since it places more stringent requirements on the nanofabrication tolerances. We recently studied the effects of changing the nanowire dimensions on the quality of fabricated SNSPDs. To quantify SNSPD quality, we used the constriction factor, which is the ratio of the current at which a nanowire ceases to have 0 resistance (the "switching current," which is limited by defects in the nanowire) to the maximum depairing current. ${ }^{16} \mathrm{~A}$ constriction factor closer to 1 is desirable for sensitivity to low-energy photons. In that particular investigation, it was found that the 
constriction factor degraded for narrower superconducting strips (widths between 200 and $55 \mathrm{~nm}$ were studied), which suggests that the edge roughness plays a significant role in limiting the switching current. In addition, it was found that increasing the temperature above $20 \%$ of the critical temperature $\left(T_{c}\right)$ also leads to a degradation in the constriction factor, shedding light on the optimum operating temperature for SNSPDs. It is expected that advances in nanostrip fabrication techniques will improve the edge roughness, improving the yield of low-energy sensitive SNSPDs. The second approach, reduction of the superconducting gap energy and thus the transition temperature, requires a lower operating temperature ( 100 to $300 \mathrm{mK})$ in order to achieve the optimum sensitivity to long wavelength photons. Reduction of the free carrier density, the third approach to improve the energy sensitivity, is the most attractive avenue to explore because it does not require a reduction of the operating temperature and yield should not be affected.

We fabricated detectors from WSi to have a lower free carrier density by increasing the silicon content of the films. The films are cosputtered from separate W and Si targets, so the composition of the films may be tuned by adjusting the relative sputtering powers. The film thickness was $\sim 3 \mathrm{~nm}$, and the superconducting transition temperature was $3 \mathrm{~K}$. Each detector is a single $10-\mu \mathrm{m}$-long nanowire instead of a large-area meander, in order to reduce the probability of fabrication defects or constrictions along the length of the wire.

The SNSPDs were measured at a temperature of $0.9 \mathrm{~K}$ and flood illuminated using various quantum cascade lasers also mounted inside the cryostat emitting at different wavelengths. Figure 2(a) shows normalized, background-subtracted photoresponse count rate (PCR) versus bias current curves for these SNSPDs. PCR curves are shown for four different nanowire widths $(50,60,70$, and $80 \mathrm{~nm})$, and measurements were obtained at four wavelengths $(1.55,4.8,7.4$, and $9.9 \mu \mathrm{m}$ ). The nanowires show the onset of a saturation plateau at a wavelength of $7.4 \mu \mathrm{m}$ for all four wire widths, but do not saturate at $9.9 \mu \mathrm{m}$. By further lowering the $T_{c}$ of the WSi film, saturated efficiency has recently been demonstrated at $9.9 \mu \mathrm{m}$ with a wire width of $50 \mathrm{~nm} .{ }^{17}$ The limits of SNSPD sensitivity to low-energy photons have not yet been reached or fully understood, thus both experimental and theoretical studies in this direction are active in the field.

As described in the introduction, the presence of a saturation plateau in the photoresponse versus bias curve is not only necessary for ultrastable operation, but also indicates $100 \%$ internal detection efficiency. The total detection efficiency of an SNSPD depends both on this internal

(a)

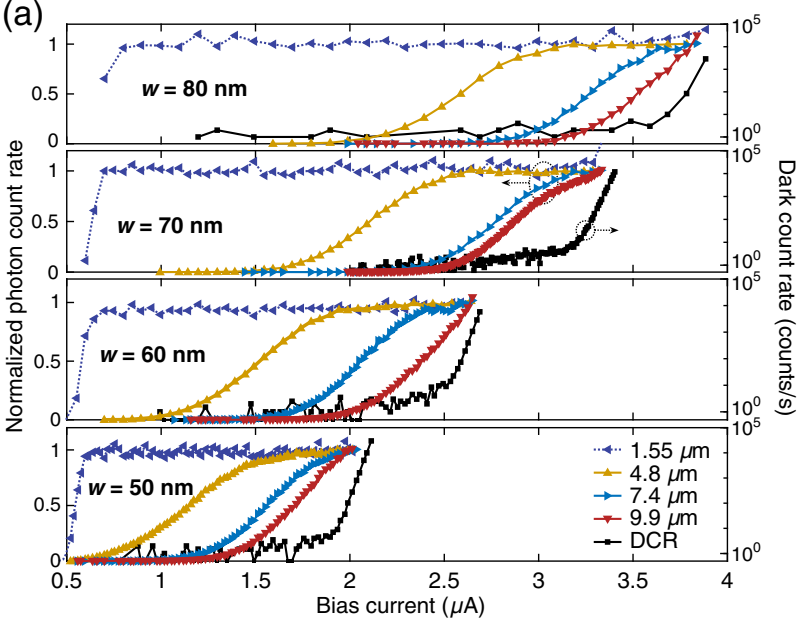

(b)
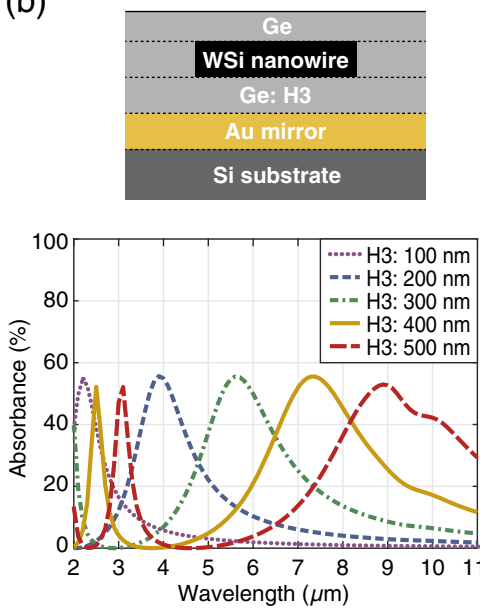

Fig. 2 (a) Normalized PCR versus bias current curves for SNSPDs fabricated from a WSi film with a higher silicon content (sputtering power on silicon target increased from 180 to $260 \mathrm{~W}$ ). PCR curves are shown for four different nanowire widths $(50,60,70$, and $80 \mathrm{~nm})$ and measurements were obtained at four wavelengths $(1.55,4.8,7.4$, and $9.9 \mu \mathrm{m})$. The SNSPDs become sensitive to longer wavelengths at higher bias currents. The efficiency is observed to saturate at $1.55,4.8$, and $7.4 \mu \mathrm{m}$ for all wire widths, but not at $9.9 \mu \mathrm{m}$. (b) Simulation of optical absorption efficiency with an optimized optical cavity. The top image shows a schematic of the cross section of a simple optical stack based around a Fabry-Pérot cavity. Light is incident from the top of the device. The bottom plot shows modeled spectra of absorption into the WSi nanowires for different thicknesses of the lower Ge layer $(\mathrm{H} 3)$. The model indicates that absorption above $50 \%$ is possible. 
efficiency and on the optical absorption into the nanowire layer. The absorption efficiency is enhanced by embedding the nanowire in an optical stack that maximizes the electric field at the nanowire. Figure 2(b) shows a schematic of a simple optical cavity stack that could be used for mid-IR detectors. A 100-nm gold layer is coated as a reflection mirror on a silicon substrate to reflect normal incident light. A 2.5-nm-thick WSi nanowire meander with 150-nm-wide wires on a 300-nm pitch is embedded in Ge layers, creating a Fabry-Pérot cavity. We utilized a rigorous coupled-wave analysis model to calculate light absorption in the layer of WSi nanowires as a function of the thickness of the bottom Ge layer. The peak absorption efficiency in WSi nanowires is $\sim 55 \%$ in the mid-IR region from 2 to $10 \mu \mathrm{m}$. Based on this simulation result, we can predict that mid-IR SNSPDs with a total detection efficiency of $\sim 50 \%$ are achievable. Higher absorption and broader spectral bandwidths may be possible with different materials or a more complex stack design. We are currently assessing additional dielectric materials for this purpose.

\subsection{Kilopixel Arrays}

The TRA-S and TRA-M bands of the MISC instrument will have a spectral resolving power of $R=50$ to 100 , which corresponds to $\sim 50$ spectral bins per band. The minimal detector array for each of these bands should have a few pixels per spectral bin and several pixels in the spatial dimension, leading to an array of 500 pixels per band. Additionally, the Origins Science and Technology Definition Team is exploring the use of an innovative densified pupil design to enhance the optical stability of MISC, ${ }^{18}$ so several subarrays will be required per wavelength band. Overall, between 1 and 10 thousand pixels will be needed at a minimum.

A variety of multiplexing schemes have been demonstrated for SNSPDs, which could be scaled to kilopixel-class arrays. ${ }^{19}$ SNSPDs are compatible with frequency domain multiplexing schemes similar to MKID readout, and 16-pixel SNSPD arrays have been demonstrated with both dc and ac biasing schemes. ${ }^{20,21}$ SNSPDs' high timing resolution and low velocity of propagation can be converted into position information by reading out the time difference between pulses leaving both ends of the device: the closer the position of the absorbed photon to one end of the nanowire, the longer the delay between when the pulse reaches the closer end of the nanowire and when it reaches the farther end of the nanowire. This time-of-flight information has been used to construct an imager with 590 effective pixels out of a single nanowire element. ${ }^{22}$ Superconducting logic has also been employed to read out SNSPD arrays, with up to 64 pixels addressed individually ${ }^{23}$ and 16 pixels multiplexed in the row-column architecture described below. $^{24}$

The most mature multiplexing scheme for SNSPDs is arguably the "row-column" approach, ${ }^{25,26}$ which we have recently demonstrated for a kilopixel SNSPD array. ${ }^{14}$ This technique allows multiplexing of $N^{2}$ pixels using only $2 N$ readout lines. The principle of operation is shown schematically in Fig. 3(a). Each row or column of the array has a bias tee to apply a dc bias current to the pixels and to read out ac signals. Bias current is introduced through each row and divided equally among the pixels in the row using a resistor in series with each pixel. The current through each pixel in the row flows out through a separate column line, where it is connected to ground through a resistor or a large inductor. When a photon is detected in a pixel, the pixel becomes resistive and diverts its current to the row readout amplifier, generating a positive pulse. The current flowing through the column corresponding to that pixel is now lower due to the pixel's diverted current, generating a negative pulse in the column amplifier. The correlation between a positive row pulse and a negative column pulse determines which pixel produced the detection event.

The $32 \times 32$ row-column array presented by Wollman et al. ${ }^{14}$ was fabricated with $30 \mu \mathrm{m}$ pixels on a $50-\mu \mathrm{m}$ pitch. The overall array size was a $1.6-\mathrm{mm}$ square, of which $960 \times 960 \mu \mathrm{m}^{2}$ consisted of active nanowire regions [Fig. 3(b)]. At its design wavelength of $1550 \mathrm{~nm}$, the array had near-saturated internal detection efficiency, as shown in Fig. 3(c). At higher bias currents, more pixels exhibit excessive count rates due to defects in the nanowires. ${ }^{27}$ Figure $3(\mathrm{~d})$ shows an example of imaging with the array biased at a current of $3 \mu \mathrm{A}$ per pixel. Four dead pixels are evident, along with two hot pixels and three suppressed pixels, giving an overall yield above 99\%. At higher bias currents, the emergence of additional hot pixels degrades the effective yield. The measurements presented in Fig. 3 were taken with a single array from the first kilopixel 
(a)

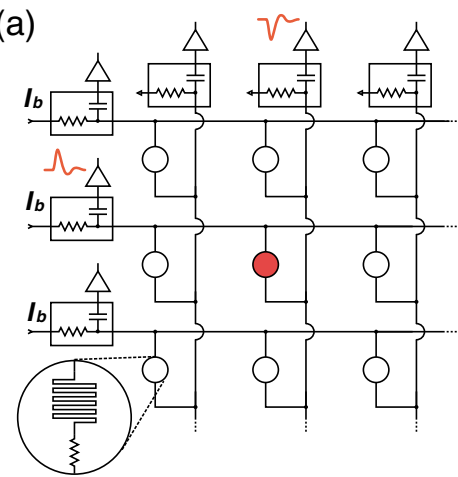

(c)

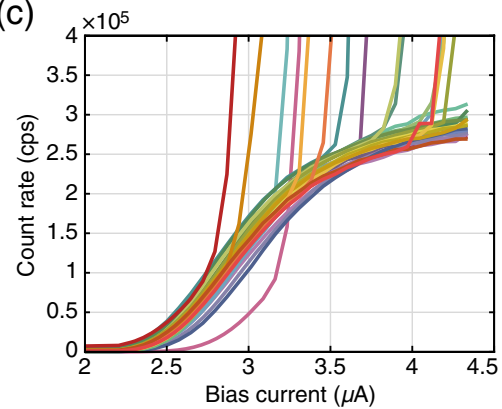

(b)

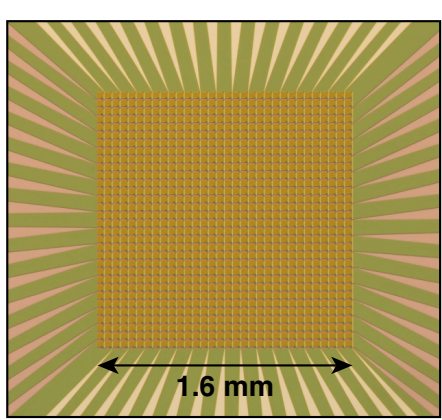

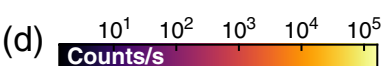

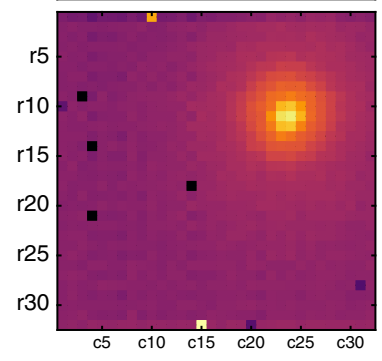

Fig. 3 (a) Row-column multiplexing architecture. When a pixel detects a photon, voltage pulses are observed on the pixel's row and column readout lines. $N^{2}$ pixels can be addressed using $2 N$ readout lines. (b) Optical micrograph of a 1024-pixel row-column SNSPD array. (c) Count rate versus average pixel bias current for each row of the kilopixel array under 1550-nm flood illumination. ${ }^{14}$ (d) Log-scale count rate image of a laser spot taken with the array biased at $3 \mu \mathrm{A}$, showing dead and hot pixels. ${ }^{14}$

fabrication run and without prior cryogenic screening to preselect devices for yield, so we expect overall yield and performance to improve with additional optimization of the fabrication process and with prescreening.

Row-column arrays are known to suffer from attenuation of their output signals due to current redistribution: when a pixel fires, some of its current is directed into other pixels in its row and column instead of through the readout amplifiers. This effect will be particularly harmful for mid-IR detectors, which have lower operating currents than detectors for shorter wavelengths. We have recently developed an alternative row-column multiplexing scheme that avoids this problem. In a "thermal row-column" (TRC) array, each row or column consists of a single long nanowire element. The rows and columns are fabricated separately on two layers with a thin dielectric spacer between them. When a photon is detected on one layer, some of the phonons produced by Joule heating of the resistive region propagate through the spacer and trigger an event on the other layer directly beneath the hotspot. Coincidences between row and column events, therefore, provide spatial information about the location of the detection. TRC arrays do not require pixel resistors to distribute the current uniformly between pixels, so they are able to achieve high optical fill factors. This scheme has been demonstrated recently in a $4 \times 4$ array optimized for $1550 \mathrm{~nm} \cdot{ }^{28}$ At sufficiently high bias currents, the thermal coupling efficiency between the two layers was found to be close to $100 \%$. Kilopixel-scale TRC arrays are currently under development, and we are investigating whether the thermal coupling can be optimized to work with the lower superconducting gap nanowires needed for mid-IR arrays.

\subsection{Large Active Areas}

The current optical design for the MISC instrument disperses light across areas of approximately $0.5 \times 5 \mathrm{~mm}^{2}$ at the focal plane for each subpupil of the TRA-S band and $\sim 1 \times 10 \mathrm{~mm}^{2}$ at the focal plane for each subpupil of the TRA-M band. ${ }^{2}$ SNSPD arrays must incorporate a large 


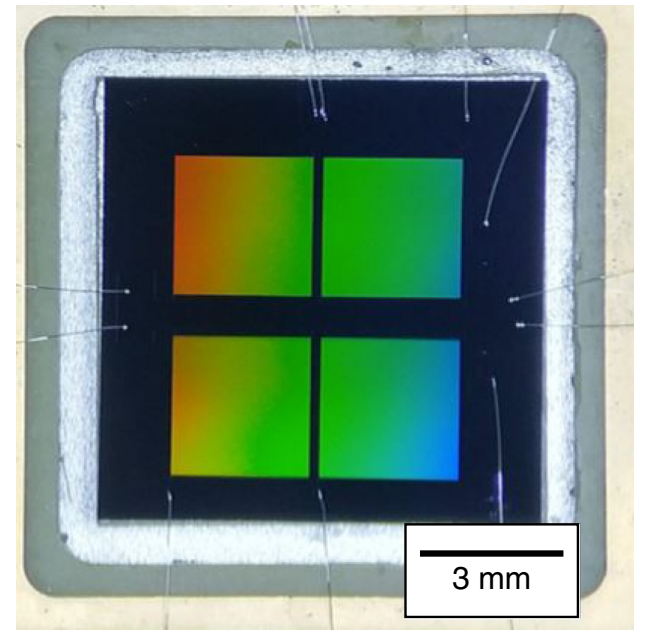

Fig. 4 Optical micrograph of several $3.1 \times 3.1 \mathrm{~mm}^{2}$ single-pixel detectors fabricated with conventional photolithography to explore SNSPD yield across larger active areas. These detectors are the largest SNSPDs fabricated to date.

length of superconducting wire to cover such active areas, whether or not it is broken up into one or many pixels. The total nanowire length is subject to the typical limitations on yield, namely: material inhomogeneity (contaminants, grain boundaries, etc.), geometrical imperfections from lithography and patterning, and topographical disruptions to the film continuity (such as particles pre-existing on the substrate prior to superconducting film deposition). However, recent developments in superconducting detector design and fabrication ${ }^{29-31}$ have enabled few-micron-wide superconducting detectors that are potentially much more robust to many of these issues. The large dimensions of these devices are amenable to fabrication with conventional photolithography, facilitating the parallel production of many devices at low cost. We have begun to explore fabrication of WSi-based single-pixel devices (wire width of $900 \mathrm{~nm}$ ) up to $3.1 \times 3.1 \mathrm{~mm}^{2}$ in area (Fig. 4) and have observed switching current densities close to the expected value required for saturated internal detection efficiency at $1550 \mathrm{~nm}$. Further testing will reveal more about the relative role of different yield factors, as well as how such detectors can be modified for longer wavelengths.

\section{Outlook for High-Stability SNSPDs}

To observe the spectral features of an exoplanet's atmosphere, Origins must observe many transits of a single star in order to resolve changes on the order of 10 to 100 parts per million in the star's spectrum. MISC, therefore, requires an unprecedented 5-ppm pixel-to-pixel stability for its 2.8 to 5.5 and 5.5 to $11 \mu \mathrm{m}$ bands $^{2}$ on timescales of an exoplanet transit (on the order of tens of minutes to several hours). Low-frequency drifts in the amplifier gain of $\mathrm{HgCdTe}$ or BIB detectors can be a limiting factor for their stability. ${ }^{3,32}$ SNSPDs exhibit a digital-like signal for each photon event and are, therefore, immune to amplifier noise. As discussed above, SNSPDs can also exhibit saturated internal detection efficiency at sufficiently high bias currents, greatly reducing the sensitivity to current drifts.

In Fig. 5, we simulate the sensitivity of an SNSPD pixel's efficiency to drifts in the applied bias current. The observed count rate is calculated as $1 / E[t]$, where $E[t]$ is the expected photon interarrival time. Following Rosenberg et al., ${ }^{9}$ the probability density for the interarrival time $t$ is $P(t) d t=e^{-R \int_{0}^{t} \eta\left(t^{\prime}\right) \mathrm{d} t^{\prime}} R \eta(t) d t$, where $R$ is the average rate of photons absorbed in the nanowire. Here, we assume that the photon arrivals follow Poisson statistics. The efficiency, $\eta(t)=\eta[I(t)]$ depends on the instantaneous bias current $I(t)$ as shown in Fig. 1(c).

Figure 5(a) shows that for low count rates, a $0.1 \%$ control on the bias current will affect the observed count rate by a negligible $\approx 0.2 \mathrm{ppm}$. This is a direct result of the small slope in the efficiency versus bias current curve at the applied bias current (flatness of the plateau). For our analysis, we assumed that the SNSPD efficiency versus bias current follows a typical error 
(a)

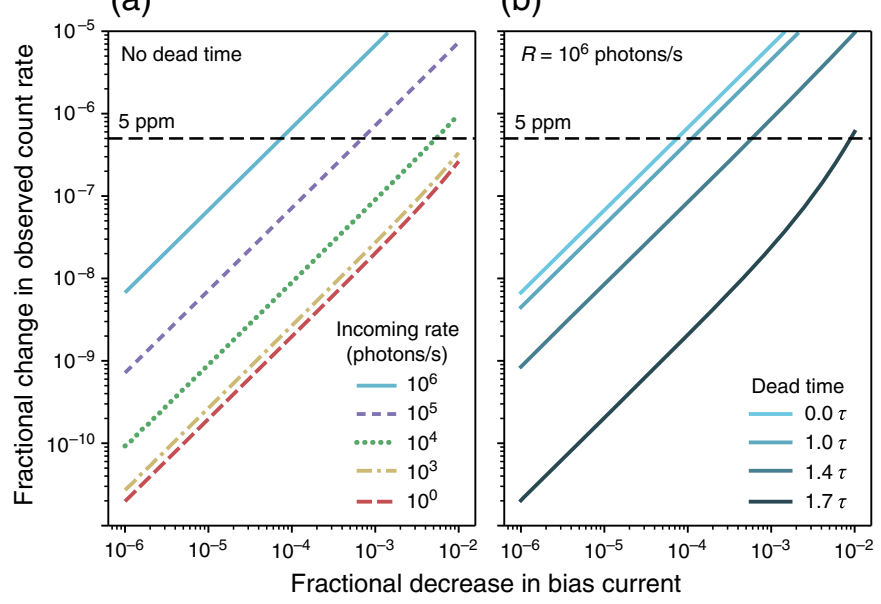

Fig. 5 Models of SNSPD photometric stability as a function of bias current, count rate, and software-enforced dead times. (a) The fractional change in observed pixel count rate versus the fractional change in applied bias current for different incoming rates of absorbed photons. At low incoming rates, the current can easily be controlled so that the observed count rate is stable to better than the required $5 \mathrm{ppm}$. (b) At high incoming rates, a software dead time can be applied to greatly improve the detector stability. The fractional change in count rate corresponds to the photometric stability. Incoming rates for a target such as TRAPPIST-1 are expected to be on the order of $10^{4} /$ photons/s/pixel, or $10^{6} /$ photons/s/row in a row-column architecture.

function curve. ${ }^{33}$ The specific curve chosen for this analysis is shown in red in Fig. 1(c), and the bias was assumed to be $85 \%$ of the switching current. As seen in Fig. 2(a), it is more difficult to achieve a wide plateau region at longer wavelengths, and so there may be increased sensitivity to current drifts as wavelength increases. In addition, nonidealities, including inhomogeneities in the wire thickness and width or a design that causes illumination of wider bend regions, can lead to a sloped PCR curve. We plan on performing precise measurements of the plateau region of SNSPDs to quantify deviations from the error function assumption.

At high count rates, the detector appears to become less stable with regards to the bias current. This is a secondary effect of the "blocking loss." During a detection event, the current in the nanowire is reduced to almost zero when it is shunted into the readout circuit. As shown in Fig. 1(b), the current returns to the nanowire exponentially $I(t)=I_{0}\left(1-e^{-t / \tau}\right)$, with a timescale $\tau=L_{\mathrm{SNSPD}} / R_{\text {load }}$ given by the nanowire inductance and readout resistance. Typically, $\tau \approx 5$ to $50 \mathrm{~ns}$ but depends on the exact detector design. At high count rates, it becomes more likely for the next photon to be absorbed in the nanowire before the current in the detector is able to fully recover. A fraction of the photon absorptions occurs during periods of low detector efficiency resulting in blocking loss. Because the time it takes the detector to recover to an efficient bias current depends on the applied bias current, the blocking loss also results in more sensitivity to drifts in the bias current.

In Fig. 5(b), we show the effects of applying a software-defined dead time in a high count rate scenario. By rejecting out all detection events that occur within $1.7 \tau$ of the previous detection, we can recover the stability exhibited by low count rate scenarios. For example, in the simulation shown in Fig. 5(b) with a photon absorption rate of $R=10^{6}$ photons/s, a $10^{-4}$ change in the bias current results in a $0.02 \mathrm{ppm}$ change in the observed count rate if we apply a $1.7 \tau=61 \mathrm{~ns}$ dead time. The decrease in global detector efficiency due to the unavoidable blocking loss at this count rate is $3.6 \%$, and the applied dead time reduces the efficiency by an additional $2.2 \%$.

In this analysis, we have only considered the effects of drifts in the bias current on the count rate of a single pixel, which is equivalent to calculating the photometric stability of a single pixel. The current-dependent pixel-to-pixel stability should be better than the photometric stability, because drifts in the bias current are likely to be correlated across the array. Other factors, such as crosstalk, may influence the pixel-to-pixel stability once SNSPDs are combined in an array, however. We are currently exploring methods of performing stability measurements in both the near- and mid-IR in order to identify any unforeseen contributions to SNSPD stability. 
Measuring detector stability on the parts-per-million level poses a challenge in itself. For example, in the shot noise limit, one must collect $10^{12}$ photons to reduce the shot noise to the partsper-million level, which corresponds to $>10$ days of counting at a rate of $10^{6}$ counts/s/pixel or $>100$ days at a rate of $10^{5}$ counts/s/pixel.

\section{Conclusion}

Although the demonstration of large-format, ultrastable, mid-IR SNSPD arrays poses a significant technological challenge, there is a clear path for development. As shown above, the required mid-IR sensitivity and necessary array scales have each been realized on a proof-of-principle level, and initial calculations of SNSPD stability are promising. The difficulties for producing large-format SNSPD arrays suitable for the MISC transit spectrometer will primarily be in maintaining high fabrication uniformity and yield while moving to the narrower wires that are necessary for mid-IR sensitivity. Further work is also necessary to explore dielectric materials that are transparent in the mid-IR for use in optical stacks to enhance the detector efficiency, as well as developing low-power readout of several thousand pixels while maintaining high count rates.

\section{Acknowledgments}

A. B. Walter's research was supported by an appointment to the NASA Postdoctoral Program at the Jet Propulsion Laboratory, administered by Universities Space Research Association under contract with NASA. Part of this research was performed at the Jet Propulsion Laboratory, California Institute of Technology, under contract with the National Aeronautics and Space Administration (Grant No. 80NM0018D0004). Support for this work was provided by the NASA ROSES-APRA program and by the Defense Advanced Research Projects Agency, Defense Sciences Office, DARPA DETECT program. The authors would also like to thank the DETECT team at MIT for their contributions to this work.

\section{References}

1. National Research Council, New Worlds, New Horizons in Astronomy and Astrophysics, The National Academies Press, Washington, DC (2010).

2. M. Meixner et al., "Origins space telescope mission concept study report," arXiv:1912.06213 (2019).

3. D. Deming et al., "Discovery and characterization of transiting super earths using an all-sky transit survey and follow-up by the James Webb Space Telescope," Publ. Astron. Soc. Pac. 121, 952-967 (2009).

4. T. P. Greene et al., "Characterizing transiting exoplanet atmospheres with JWST," Astrophys. J. 817, 17 (2016).

5. B. Cabrera et al., "Detection of single infrared, optical, and ultraviolet photons using superconducting transition edge sensors," Appl. Phys. Lett. 73(6), 735-737 (1998).

6. O. Noroozian et al., "Enabling technologies for photon-counting spectroscopy with the Origins Space telescope (OST) in the mid/far-infrared region (Conference Presentation)," Proc. SPIE 10708, 107080Q (2018).

7. D. V. Reddy et al., "Exceeding $95 \%$ system efficiency within the telecom C-band in superconducting nanowire single photon detectors," in Conf. Lasers and Electro-Opt., Optical Society of America, p. FF1A.3 (2019).

8. Y. Hochberg et al., "Detecting sub-GeV dark matter with superconducting nanowires," Phys. Rev. Lett. 123, 151802 (2019).

9. D. Rosenberg et al., "High-speed and high-efficiency superconducting nanowire single photon detector array," Opt. Express 21, 1440-1447 (2013).

10. F. Marsili et al., "Mid-infrared single-photon detection with tungsten silicide superconducting nanowires," in Conf. Lasers and Electro-Opt. 2013, Optical Society of America, p. CTu1H.1 (2013). 
11. L. Chen et al., "Ultra-sensitive mid-infrared emission spectrometer with sub-ns temporal resolution," Opt. Express 26, 14859-14868 (2018).

12. V. B. Verma et al., "Towards single-photon spectroscopy in the mid-infrared using superconducting nanowire single-photon detectors," Proc. SPIE 10978, 109780N (2019).

13. M. Shaw et al., "Superconducting nanowire single photon detectors for deep space optical communication (Conference Presentation)," Proc. SPIE 10096, 100960J (2017).

14. E. E. Wollman et al., "Kilopixel array of superconducting nanowire single-photon detectors," Opt. Express 27, 35279-35289 (2019).

15. T. Matsuo et al., "Photometric precision of a si:as impurity band conduction mid-infrared detector and application to transit spectroscopy," Publ. Astron. Soc. Pac. 131, 124502 (2019).

16. S. Frasca et al., "Determining the depairing current in superconducting nanowire singlephoton detectors," Phys. Rev. B 100, 054520 (2019).

17. V. B. Verma et al., "Single-photon detection in the mid-infrared up to 10 micron wavelength using tungsten silicide superconducting nanowire detectors," arXiv:2012.09979 (2020).

18. T. Matsuo et al., "A new concept for spectrophotometry of exoplanets with space-borne telescopes," Astrophys. J. 823, 139 (2016).

19. A. N. McCaughan, "Readout architectures for superconducting nanowire single photon detectors," Supercond. Sci. Technol. 31, 040501 (2018).

20. S. Doerner et al., "Frequency-multiplexed bias and readout of a 16-pixel superconducting nanowire single-photon detector array," Appl. Phys. Lett. 111(3), 032603 (2017).

21. A. Sinclair et al., "Demonstration of microwave multiplexed readout of DC-biased superconducting nanowire detectors," IEEE Trans. Appl. Supercond. 29, 2200704 (2019).

22. Q.-Y. Zhao et al., "Single-photon imager based on a superconducting nanowire delay line," Nat. Photonics 11(4), 247-251 (2017).

23. S. Miyajima et al., "High-time-resolved 64-channel single-flux quantum-based address encoder integrated with a multi-pixel superconducting nanowire single-photon detector," Opt. Express 26, 29045-29054 (2018).

24. M. Yabuno et al., "Scalable implementation of a superconducting nanowire single-photon detector array with a superconducting digital signal processor," Opt. Express 28, 1204712057 (2020).

25. V. B. Verma et al., "A four-pixel single-photon pulse-position array fabricated from WSi superconducting nanowire single-photon detectors," Appl. Phys. Lett. 104(5), 051115 (2014).

26. M. S. Allman et al., "A near-infrared 64-pixel superconducting nanowire single photon detector array with integrated multiplexed readout," Appl. Phys. Lett. 106(19), 192601 (2015).

27. A. J. Kerman et al., "Readout of superconducting nanowire single-photon detectors at high count rates," J. Appl. Phys. 113(14), 144511 (2013).

28. J. P. Allmaras et al., "Demonstration of a thermally coupled row-column snspd imaging array," Nano Lett. 20(3), 2163-2168 (2020).

29. Y. P. Korneeva et al., "Different single-photon response of wide and narrow superconducting strips," Phys. Rev. Appl. 13, 024011 (2020).

30. J. Chiles et al., "Superconducting microwire detectors with single-photon sensitivity in the near-infrared," Appl. Phys. Lett. 116, 242602 (2020).

31. I. Charaev et al., "Large-area microwire MoSi single-photon detectors at $1550 \mathrm{~nm}$ wavelength," Appl. Phys. Lett. 116, 242603 (2020)..

32. M. E. Ressler et al., "The mid-infrared instrument for the James Webb Space Telescope, VIII: the MIRI focal plane system," Publ. Astron. Soc. Pac. 127, 675-685 (2015).

33. A. G. Kozorezov et al., "Fano fluctuations in superconducting-nanowire single-photon detectors," Phys. Rev. B 96, 054507 (2017).

Biographies of the authors are not available. 